\title{
Childhood-onset autosomal recessive slowly progressive spinocerebellar ataxia
}

INSERM

\section{Source}

INSERM. (1999). Orphanet: an online rare disease and orphan drug data base. Childhoodonset autosomal recessive slowly progressive spinocerebellar ataxia. ORPHA:284324

A rare, genetic, autosomal recessive cerebellar ataxia disease characterized by slowly progressive spinocerebellar ataxia developing during childhood, manifesting with gait and limb ataxia, postural tremor, dysarthria, sensory alterations (e.g. decreased vibration sense), eye movement anomalies (i.e. nystagmus, saccadic pursuit, oculomotor apraxia), upper and lower limb fasciculations, and hyperreflexia with Babinski signs. Brain imaging reveals cerebellar, pontine, vermian and medullar atrophy. 